\title{
Wavelength response of a surface plasmon resonance palladium-coated optical fiber sensor for hydrogen detection
}

\author{
Cedric Perrotton \\ Université de Strasbourg \\ Laboratoire des Systèmes Photoniques \\ Pole API, Bvd Sébastien Brant \\ 67400 Illkirch, France
}

\author{
Martin Slaman \\ Vrije Universiteit \\ Faculty of Sciences \\ Department of Physics and Astronomy \\ Condensed Matter Physics \\ De Boelelaan 1081 \\ 1081 HV Amsterdam, The Netherlands \\ and \\ Delft University of Technology \\ Department of Chemical Engineering \\ Julianalaan 136 \\ 2628 BL Delft, The Netherlands
}

Nicolas Javahiraly

Université de Strasbourg

Laboratoire des Systèmes Photoniques

Pole API, Bvd Sébastien Brant

67400 Illkirch, France

\section{Herman Schreuders}

Bernard Dam

Delft University of Technology

Department of Chemical Engineering

Julianalaan 136

2628 BL Delft, The Netherlands

\section{Patrick Meyrueis}

Université de Strasbourg

Laboratoire des Systèmes Photoniques

Pole API, Bvd Sébastien Brant

67400 Illkirch, France

\begin{abstract}
An optical fiber using palladium as sensitive layer is characterized in the range of 450 to $900 \mathrm{~nm}$. The sensitive layer is deposited on the outside of a multimode fiber, after removing the optical cladding. The sensor is based on a measurement technique that uses the surface plasmon resonance effect. A continuous change in output intensity is observed as a function of the hydrogen concentration between $0.5 \%$ and $4 \% \mathrm{H}_{2}$ by volume in Argon. The response shows that the transmitted intensity can either decrease or increase, depending on the selected wavelength. This behavior is directly related to the change in reflectance upon hydrogenation between the polarization $s$ and $p$. The loading time is $30 \mathrm{~s}$ and the unloading time is $90 \mathrm{~s}$ in a mix of argon and $10 \%$ of oxygen. The detectors show a good reproducibility. (C) 2011 Society of Photo-Optical Instrumentation Engineers (SPIE). [DOI: 10.1117/1.3529432]
\end{abstract}

Subject terms: optical sensors; fiber optic sensors; surface plasmon resonance; hydrogen sensors; palladium.

Paper 100522PR received Jun. 25, 2010; revised manuscript received Oct. 26, 2010; accepted for publication Nov. 11, 2010; published online Jan. 28, 2011.

\section{Introduction}

Hydrogen is currently used in chemical industrial production processes as a hydrogenating agent and in aerospace industries as a fuel source. In addition, environmental and (socio-) economic issues suggest that hydrogen will become a competitive energy carrier in the near future. Its application will spread from the automobile industry to domestic products. The use of hydrogen as an energy carrier will considerably increase the hydrogen production volume and the related distribution network. Hydrogen sensors are essential to monitor hydrogen concentrations, to detect leaks, and to prevent fire and explosions. The lower flammability point of hydrogen

0091-3286/2011/\$25.00 @ 2011 SPIE is $4 \%$ in air and $74.5 \%$ for the upper limit. Currently electrochemical, thermal conductivity, catalytic, and electrical sensors are mainly used for hydrogen detection. They show in general a high sensitivity and a relativly fast response; however they are not suitable for use in an explosive environment due to the risk of ignition (hydrogen ignition energy: $0.02 \mathrm{~mJ}$ ) due to the presence of electrical current leads. Although optical fibers sensors are not yet completely mature and still require significant efforts, they appear very promising devices for hydrogen leak detection. Their geometrical configuration (small dimensions, light weight, geometrical versatility), their high electrical isolation, their simplicity of design, and especially their ability to operate in potentially explosive environments made them very suitable for hydrogen sensing. 
Most optical hydrogen sensors use a palladim (Pd) thin film layer as a catalyst agent and also as a transducer due to its high solubility to $\mathrm{H}_{2}$. The electrical, optical properties, and the lattice constant of Pd change when it interacts with hydrogen. This change is reversible and nonlinear. It is related to the Pd pressure isotherm. Numerous fiber sensor configurations have been studied in order to improve the sensitivity (especially in the low pressure range, where the solubility of hydrogen in the Pd alpha phase is low). Some of them are based on elasto-optic effects such as interferometric sensors. ${ }^{1,2}$ Others use the decrease of the complex dielectric permittivity as micro mirror sensors or evanescent wave sensors. ${ }^{3}$ Lately, fiber bragg gratings ${ }^{4-6}$ have been used, they act as a pure strain sensor due to the expansion of the Pd. Most of the optical fiber sensors present a good sensitivity but are mainly limited to the response time and the cross sensitivity. Villatoro et al. ${ }^{7}$ propose a nano tapered fiber sensor coated with ultra thin film in order to limit the diffusion time and therefore the response time. However this kind of sensor is very fragile. Multimode fiber sensors based on surface plasmon resonance (SPR) may be an alternative to conceive a robut sensor, in keeping a high sensitivity.

Here, we start from the optical surface plasmon resonance sensor introduced by Bevenot et al. using a thin Pd layer as the transducer. In Bevenot et al.' ${ }^{8}$ paper the hydrogen concentration is detected by measuring the change in intensity for a specific light input angle and wavelength. Only the transverse magnetic (TM) polarization (magnetic field perpendicular to incidence plane) is considered. We propose here to study the response when all modes are equally excited in the optical fiber. The variation of the total intensity transmitted [sum of the transverse electric (TE) (electric field perpendicular to incidence plane) and TM polarization] is then measured as an estimate for the hydrogen concentration. The advantage is to conceive a low cost sensor easily integrable and offering the possibility of a multi-point detection in a single fiber. We focus on the spectral response of the sensor. Our aim is to demonstrate the role of the change in real and in complex part of the Pd dielectric permittivity on the SPR peak. Moreover since the SPR occurs only for the TM polarization, we will see if the TE polarization is active on the sensitivity of the sensor. The response time and the reproducibility of the sensor response are also presented, as well as the effect of $\mathrm{O}_{2}$ to validate the possibility of using this sensor for safety applications.

\section{Principle}

In 1993 Chadwick et al. ${ }^{9}$ have demonstrated the SPR effect in a Pd layer in the Kretschmann configuration ${ }^{10}$ in order to realize a hydrogen sensor. Most SPR sensors are based on the change in the dielectric permittivity of the medium surrounding the metallic layer. In the case of Pd, the complex permittivity of the metallic layer itself changes with the absorption of hydrogen. The resonance angle of $\mathrm{Pd}$ on a glass substrate is $53 \operatorname{deg}$ for $\theta$, where $\theta$ is the angle between the normal and the incindent light as shown in Fig. 1. This angle cannot be reached by using an optical fiber configuration. However, Bevenot et al. ${ }^{8}$ have demonstrated that the SPR effect is still sufficient for hydrogen detection, in this configuration, due to the large width of the Pd SPR peak. The sensor consisted of a Pd layer deposited on the fiber core, over a section of the fiber, as indicated by $\mathrm{L}$ in Fig. 2. The light TM-polarized is injected into the fiber with

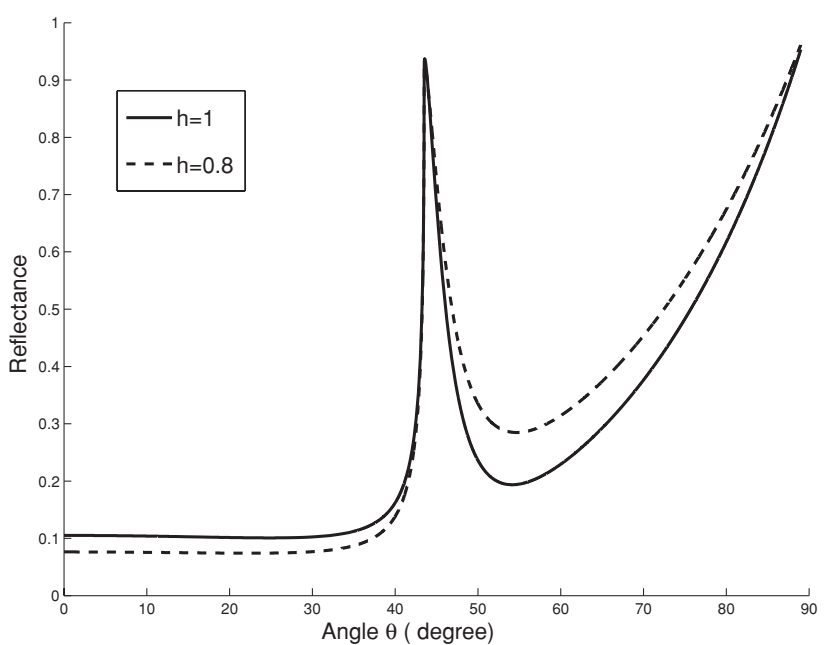

Fig. 1 Reflectance of a Pd layer with a thickness of $12 \mathrm{~nm}$ onto glass substrate as a function of the angle $\theta$. The solid and dashed lines represent the metallic state and the hydrogenated state, respectively. The permittivity of $\mathrm{Pd}$ hydride is described as $\epsilon \mathrm{Pd}, c\left(\% \mathrm{H}_{2}\right)$ $=h(c \%) \times \epsilon \mathrm{Pd}, 0 \% \mathrm{H}_{2}$, where $\epsilon \mathrm{Pd}, 0 \% \mathrm{H}_{2}=-7.64+j 8.45$ is the dielectric permittivity of the pure palladium. ${ }^{8}$ At room condition, $h=1$ and $h$ $=0.8$ mean approximately a concentration of $0 \%$ and $4 \% \mathrm{H}_{2}$, respectively.

an appropriate angle of incidence and for a given wavelength. Thus the corresponding mode bunch is excited through the fiber. The change in the transmitted intensity allows to measure the $\mathrm{H}_{2}$ concentration. However, this technique is not really convenient for applications due to the mode conversion along the fiber (and demands to maintain the polarization along the fiber). Due to the fiber impurities or the mechanical constraints, the power distribution for the mode along the fiber can be disturbed and then converted into other modes, especially when the fiber is used in an operative field environment. That is why we present here the hydrogen response when all modes are equally excited in the optical fiber for both TE and TM polarizations. The measured response is then a function of the numerical aperture shift of the sensitive section. Since multimode fibers are used, the sensor response is modeled and simulated by combining the theory of local plane waves and a geometric optics approach. The reflectance of the multilayer structure is first calculated as a function of angle by using the Fresnel coefficients with the transfer matrix method. ${ }^{11}$ The multilayer structure consists here of an infinite layer of silica, covered by a layer of Pd with a certain thickness and an infinite layer of air. The silica refractive index as a function of the wavelength is given by the Sellmeier equation

$$
n(\lambda)=P_{1}+P_{2} \lambda^{2}+P_{3} \lambda^{-2}+P_{4} \lambda^{-4}+P_{5} \lambda^{-6}+P_{6} \lambda^{-8},
$$

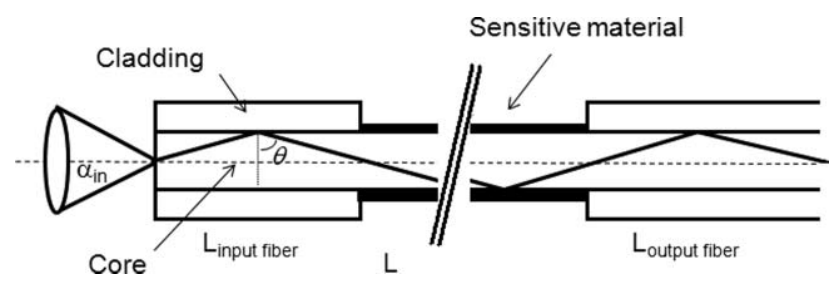

Fig. 2 Sensitive material deposited on the fiber core. 
where the coefficient $P_{1}, P_{2}, P_{3}, P_{4}, P_{5}$, and $P_{6}$ are the Schott coefficient, industry standard coefficient as determined by Schott for use with their glasses. The value of coefficients are $P_{1}=1.45179, P_{2}=-0.00365, P_{3}=-0.00243, P_{4}=$ $0.00012, P_{5}=-4.605 \times 10^{-6}$, and $P_{6}=9.635 \times 10^{-8}$ and $\lambda$ denotes the wavelength measured in $\mu \mathrm{m}$. Equation (1) is valid for the studied wavelength range. For simulating the sensor response, we need the dielectric permittivity of Pd and of Pd hydride. As reported by Sullivan, ${ }^{12}$ the dielectric permittivity data of a dc-sputtered Pd, especially the imaginary part, is very sensitive to sample preparation and measurement technique. The possible contamination or oxide overlayers on the thin film can introduce significant errors. We decided to use the dielectric permittivity of Pd obtained by von Rottkay et al. ${ }^{13}$ from ellipsometry measurements. The sample was sputtered in a high vacuum deposition facility as in our case. The thickness of the von Rottkay 's samples corresponds to the thickness range of our Pd film. For the dielectric permittivity of Pd hydride, two ways were considered. First, the data from von Rottkay is used. Second, the effect of the absorption of hydrogen on the dielectric permittivity of palladium is approximated by the following relation: ${ }^{8}$

$\epsilon_{\mathrm{Pd}, c(\%) \mathrm{H}_{2}}=h[c(\%)] \times \epsilon_{\mathrm{Pd}, 0 \% \mathrm{H}_{2}}$,

where $\epsilon_{\mathrm{Pd}, 0 \% \mathrm{H}_{2}}$ is the dielectric permittivity of pure palladium and $h$ represents a nonlinear relation with hydrogen concentration $c(\%) . h=1$ and $h=0.8$ represent a concentration of $0 \%$ and $4 \%$ at room condition, respectively. This description of $\mathrm{H}_{2}$ absorption is compared in the following with the experimental dielectric permittivity of palladium hydride obtained at $10^{5} \mathrm{~Pa}$ hydrogen pressure from von Rottkay et al. ${ }^{13}$

The transmitted intensity through the fiber is then calculated by summing the power loss/gain at each reflection over the sensitive area. The transmitted intensity $I\left(\alpha_{\text {out }}\right)$ for an incidence angle $\alpha_{\text {in }}$ is written as

$I\left(\alpha_{\text {out }}\right)=1 / 2\left[\left|r_{p}(\theta)\right|^{2 N}+\left|r_{s}(\theta)\right|^{2 N}\right] I_{0}\left(\alpha_{\text {in }}\right)$

with

$N=L /(D \times \tan \theta)$,

where $L, D, N$, and $r_{p}$ and $r_{s}$ are the sensitive length, the fiber diameter, the reflection number along the sensitive area, and $r_{p}$ and the reflection coefficient for polarization TE and TM, respectively. Only meridian rays are considered. The model does not take into account the scattering from the roughness of the thin film and the effect of mode coupling. Furthermore the TE and TM polarization are assumed to be equally distributed.

\section{Experimental Details}

The fiber used for the experiment is a multimode fiber, Newport $200 \mu \mathrm{m}$ core $/ 230 \mu \mathrm{m}$ cladding, with a numerical aperture of 0.37 . The fiber is made of pure silica for the core, a harder polymer for the cladding, and a tefzel jacket. The jacket is locally removed by mechanical stripping and the optical cladding is removed with a flame torch over a length of $2 \mathrm{~cm}$. The sensor response increases as the optically sensitive length $L$ along the fiber increases. The length $L$ is limited to $2 \mathrm{~cm}$ in order to handle the fiber without breaking it. The fiber is maintained to a support to avoid bending. After burning the cladding the core is cleaned with isopropyl alcohol (IPA), using lint free tissue, to remove the carbon residue.

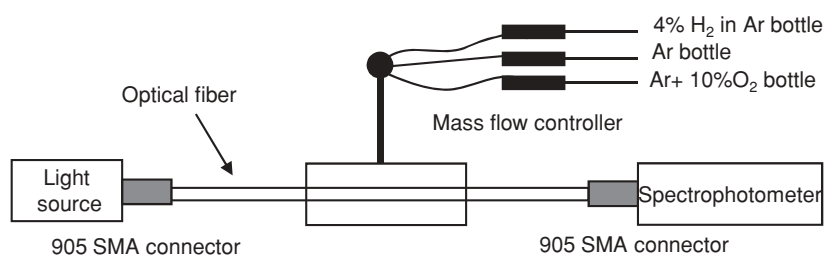

Fig. 3 Measurement setup used for hydrogen detection tests.

The fiber with a hard polymer cladding is chosen against a glass cladding type to allow an easy removing of the cladding without the need an hydrofluoric etching step. However, after this process in some samples a polymer film is left on the core. In order to obtain a clean interface the surface has to be investigated with an appropriate optical microscope. The deposition of the Pd hydrogen indicator layer is done in an AJA magnetron sputtering apparatus. The fiber is vertically set in the center of the device above the gun with a tilt angle. Due to this configuration, the deposition results in a slight gradient of the thickness along the fiber. For a deposition over a width of $2 \mathrm{~cm}$ along the fiber, the thickness varies from 9 to $14 \mathrm{~nm}$. The background pressure was $5 \times 10^{-7}$ mbar and the sputter pressure was $3 \mu$ bar argon (Ar). Palladium was sputtered at an average rate of $0.15 \mathrm{~nm} / \mathrm{s}$ using $100 \mathrm{~W}$ dc power supply. The deposition rate, as measured with a quartz crystal monitor, is confirmed afterward by a Detak profilometer. The measurement setup is depicted in Fig. 3. A tungsten halogen light source and an Ocean Optics HR 4000 spectrometer are used as a source and a detector, respectively. The fiber containing the hydrogen detector is connected via 905 SMA connectors to the source and the detector. The numerical aperture (NA) of the source is superior to the fiber in order to excite all of the fiber modes. The spectrometer is limited by a numerical aperture of 0.22 . This limitation does not disturb our measurements when the Pd layer is deposited onto the fiber core over a length of $2 \mathrm{~cm}$. The fiber NA with the sensitive section obtained experimentally is comparable. The NA is theoretically larger, 0.3 for a Pd $10 \mathrm{~nm}$ deposit over 2-cm lengths. However, the transmitted intensity, for the ray propagating between the theoretical and experimental critical angle, is less than $1 \%$ of the total intensity. In the following,

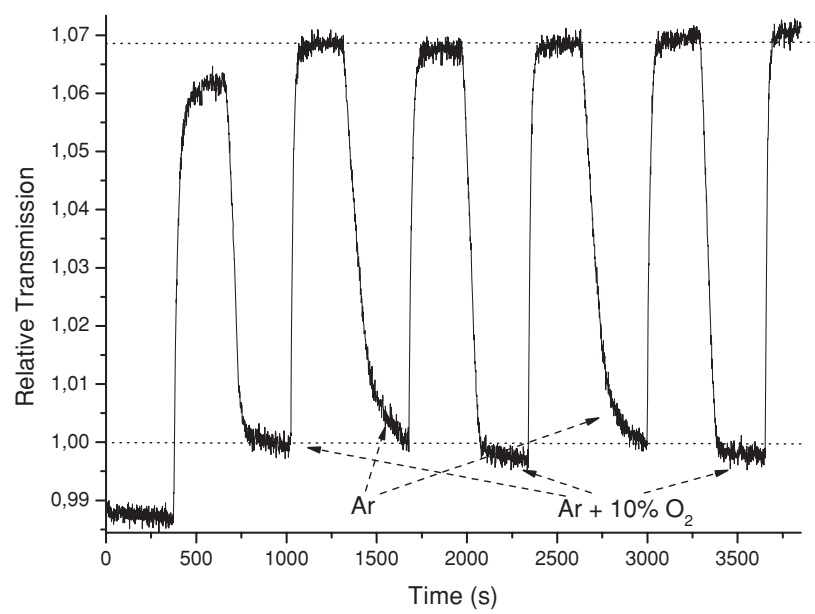

Fig. 4 Sensor response reproducibility for a concentration of $4 \%$ $\mathrm{H}_{2}$ in $\mathrm{Ar}$ at a wavelength of $849.96 \mathrm{~nm}$. Unloading are successively repeated in $\mathrm{Ar}+10 \% \mathrm{O}_{2}$ and in $\mathrm{Ar}$. 
we limit the NA to 0.22 corresponding to the spectrometer in order to compare the experimental results with the prediction. The fiber is measured in a gas cell. The gas cell is flushed with argon that contains a chosen concentration hydrogen (up to $4.4 \%$ ) and oxygen (up to $10 \%$ ). Flow rates of each gas were individually controlled with mass flow controllers. The total flow is kept at $250 \mathrm{ml} / \mathrm{min}$. All measurements are performed at atmospheric pressure and room temperature. The gas cell is purged with argon for several minutes before all measurements in order to clean the tubing of the gas system. The relative transmission is defined as the intensity measured $I_{\mathrm{H}_{2}}$, divided by the intensity measured without hydrogen $I$. The loading and unloading time is defined as the response time, needed to reach $90 \%$ of steady-state.

\section{Experimental Results and Discussion}

From Figs. 4 and 5(b) it can be concluded that the sensor response is reproducible after the first cycle. A slight drift appeared over cycles, however it seems to mainly come from the effects of pollutants onto the Pd surface rather than the formation of microcracks over time, as discussed in Sec. 3. This drift observed in Fig. 5(b) is weaker than in Fig. 4 where the unloading conditions are identical over the cycles. The sensitivity study is therefore done after one cycle. The Pd film needs at least one cycle in order to stabilize the microstructure, and to remove most poisonous elements from the Pd surface, which are gathered by transport of the sample through air.

A continuous change in intensity is observed as a function of the hydrogen concentration between $0.5 \%$ and $4 \%$ $\mathrm{H}_{2}$ in argon (Ar), as depicted in Fig. 5. During the hydrogenation the transmitted intensity through the fiber core decreases below a specific wavelength and increases above it. As a result, the response shows a wavelength dependence that was not observed in previous works. There is no significant wavelength dependence when the relative transmission is simulated with the dielectric permittivity following relation (2). Apparently, relation (2) is not satisfied in the design we use because the real and the imaginary part of the dielectric permittivity of the $\mathrm{Pd}$ hydride cannot be linked by the same constant $h$ as will be discussed later. From Fig. 6, we find that the simulated sensor response shows a similar spectral behavior as when the experimental dielectric function is used; the intensity can decrease or increase depending on the selected wavelength. Compared to the experimental results, the sensitivity of the simulated response is slightly different. The sensitivity is defined as equal to $\left|1-I_{\mathrm{H}_{2}} / I\right| \%$. The minimum sensitivity simulated is obtained at the following wavelengths: 752 and $909 \mathrm{~nm}$, for thicknesses of 9 and $14 \mathrm{~nm}$, respectively. The maximum sensitivity is obtained in the blue wavelength. Its value is $11.5 \%$ and $15.5 \%$ for a thickness of 9 and 14 nm, respectively. From Fig. 5(b), we see that the response with $4 \%$ of $\mathrm{H}_{2}$ in $\mathrm{Ar}$ shows a minimum sensitivity of $0 \%$ around $600 \mathrm{~nm}$ and a maximum of $8 \%$ at $900 \mathrm{~nm}$. The difference with the simulated results probably comes from the roughness and the thickness gradient of our sample. Moreover Vargas et al. pointed out that the optical constants of the $\mathrm{Pd}$ films due to $\mathrm{H}_{2}$ absorption depends on the nature of the substrate. ${ }^{14}$ Since in our sample the Pd is deposited on glass, the change in optical constants upon hydrogenation may be smaller than those obtained from von Rottkay. Nevertheless, it is worth noting that Yamada et al. have recently obtained the same optical constants for a Pd deposit onto a glass substrate. ${ }^{15}$ The microstructure of the Pd may also play a role on the wavelength dependence. Figure 5(a) shows the relative transmission when the sputtering pressure is set at $40 \mu$ bar during the first $10 \mathrm{~s}$ of the Pd deposition. The sensor response on loading with $4 \%$ of $\mathrm{H}_{2}$ in $\mathrm{Ar}$ shows a change with a minimum of $0 \%$ around $670 \mathrm{~nm}$ and a maximum of $13 \%$ at $450 \mathrm{~nm}$. So the sensor response appears to depend on the deposition conditions and is therefore related to the microstructures. However, the wavelength shift in the sensor response may be amplified by the surface state of the fiber before deposition. Further investigations have to be done to verify this influence. Nevertheless, the data from von Rottkay appears to be relevant to study the sensor response.

The relative transmission is simulated for the $p, s$, and for both polarizations, as represented in Fig. 7. For $p$ polarization, the transmitted intensity is increased upon hydrogenation. The sensitivity is smaller at shorter wavelength and increases toward the infrared wavelength. For $s$ polarization, the transmitted intensity is decreased upon hydrogenation. The sensitivity is larger at shorter wavelength and decreases

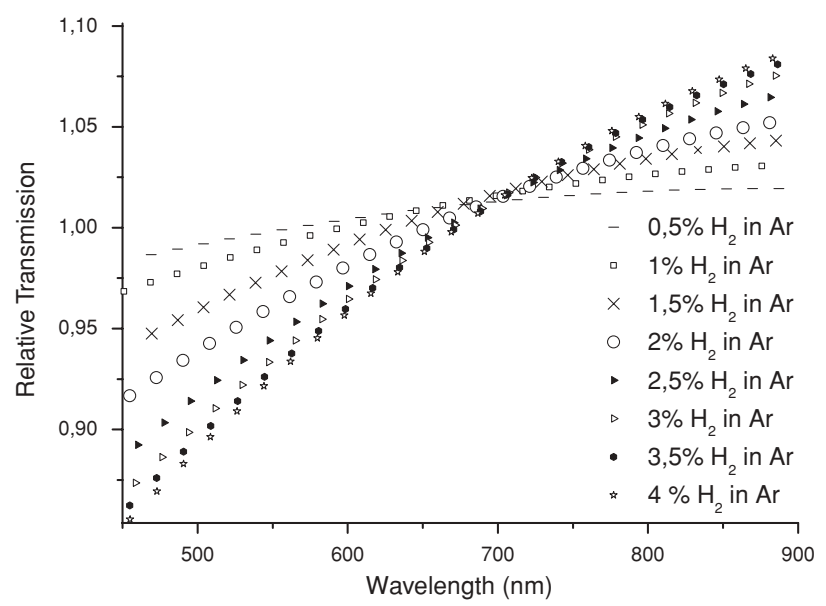

(a)

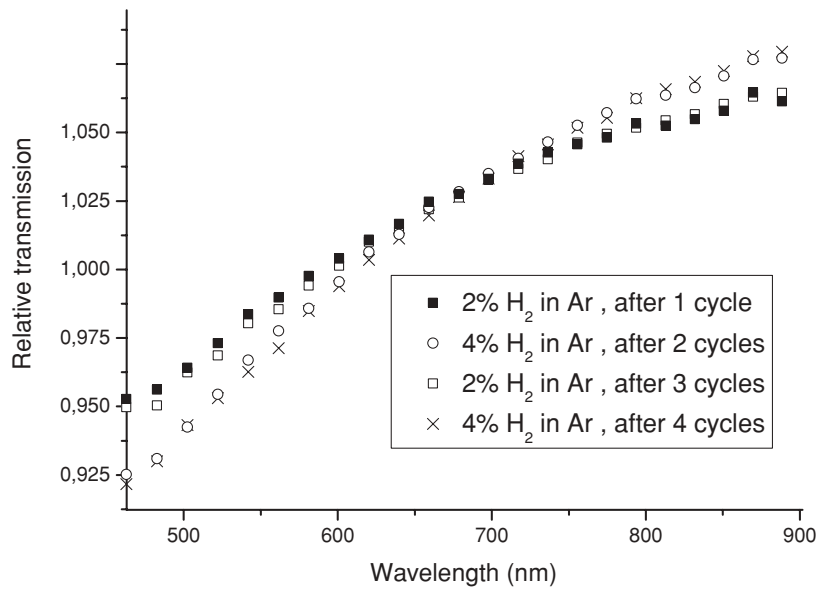

(b)

Fig. 5 Relative transmission as a function of the wavelength for different hydrogen concentrations. (a) Shows the quantitative measurement with the sensor and (b) shows the reproducibility of the measurement with the sensor. The sensor consists of a Pd layer with a thickness gradient from 9 to $14 \mathrm{~nm}$ deposited on the fiber core over a length of $2 \mathrm{~cm}$. The sputter pressure is set at $3 \mu$ bar for (b) and is set at $40 \mu$ bar during the first $10 \mathrm{~s}$ of the Pd deposition for (a). 


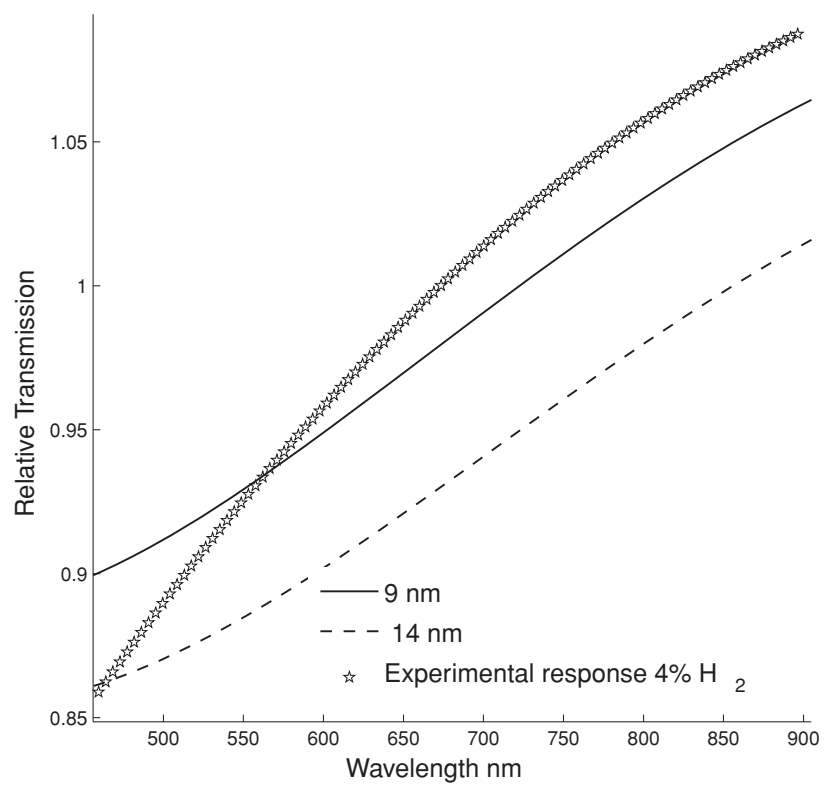

Fig. 6 Relative transmission as a function of the wavelength. Stars represent the experimental data for $4 \% \mathrm{H}_{2}$ in Ar. The solid and dashed lines represent the simultated relative transmission for 9 and $14 \mathrm{~nm}$ $\mathrm{Pd}$, respectively, deposited over a length of $2 \mathrm{~cm}$. The dielectric permittivity of Pd and Pd hydride from von Rottkay et al. ${ }^{13}$ is used.

toward the infrared wavelength. The change in reflectance depends, finally, on the wavelength for both polarizations.

To explain this behavior for both $p$ and $s$ polarization, the reflectance is calculated for different wavelengths and is represented in Fig. 8. For our study, only the angles within the fiber NA are considered. Owing to hydrogen absorption, the real $\epsilon_{1}$ and the imaginary part $\epsilon_{2}$ of the dieletric permittivity decrease. This can be explained by an increase of the inelastic electron collision frequency and a decrease of the electron plasma frequency in the Drude model. According to von Rottkay's data ${ }^{13}$ the change in $\Delta \epsilon_{1}=\left|\epsilon_{1}(P d H x)-\epsilon_{1}(P d)\right|$ and $\Delta \epsilon_{2}=\left|\epsilon_{2}(P d H x)-\epsilon_{2}(P d)\right|$ depends on the wavelength.

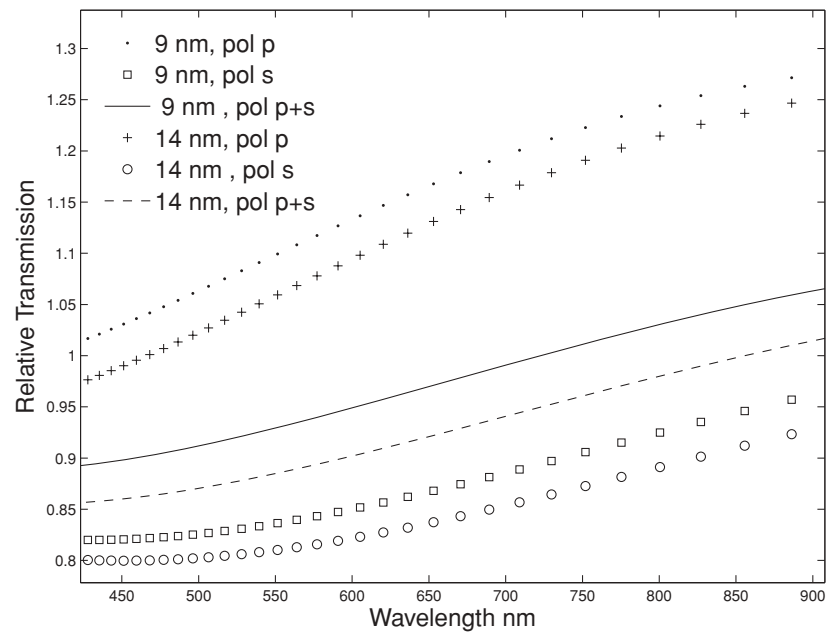

Fig. 7 Relative transmission as a function of the wavelength for different thickness. Square/circle, dotted/plus, and solid/dashed lines represent the response for $s, p$, and the sum of both polarizations. The relative transmission is simulated by using the dielectric permittivity of Pd hydride (from von Rottkay et al. ${ }^{13}$ ).
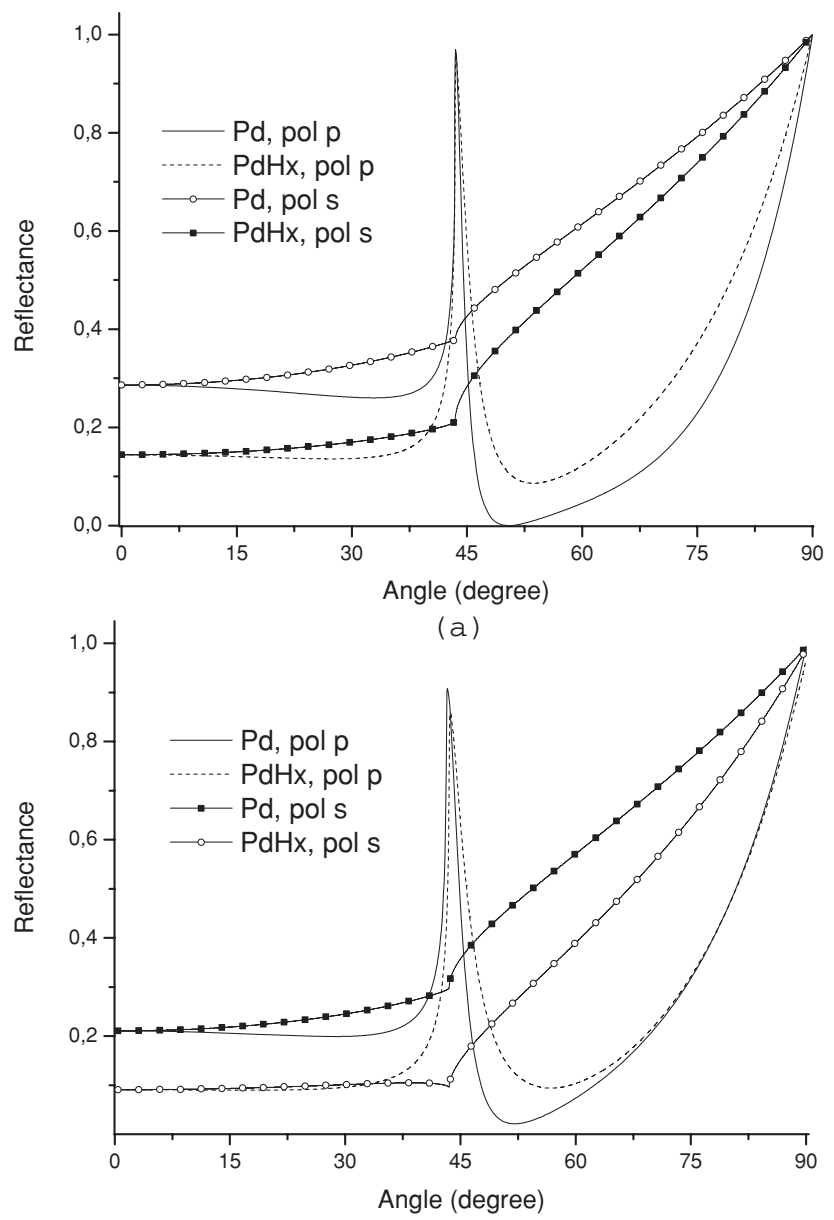

(b)

Fig. 8 Reflectance of a Pd layer and a PdHx layer of $12 \mathrm{~nm}$ onto glass substrate as a function of the angle $\theta$ ( 0 deg corresponds to the normal incidence). The dielectric permittivity of pure and hydride palladium (from von Rottkay et al. ${ }^{13}$ ) are used. The wavelength is (a) $800.45 \mathrm{~nm}$ and (b) $468 \mathrm{~nm}$.

Both of them are increasing toward the infrared wavelength and are stronger for $\Delta \epsilon_{2}$. The value of $\Delta \epsilon_{1}$ and $\Delta \epsilon_{2}$ are 5.546 and 1.385 at $468 \mathrm{~nm}$ and 8.900 and 12.543 at $992 \mathrm{~nm}$.

We now consider the reflectance for $p$ polarization. It is well known that the Pd presents a broad SPR peak. The Pd is a highly absorbing metal, $\epsilon_{2} \approx \epsilon_{1}$. Upon hydrogenation, the width of the SPR peak decreases. As the Pd hydride is less absorbing, the propagation length of the SP wave is longer. The smaller $\epsilon_{2}$ is as compared to $\epsilon_{1}$, the thinner the SPR peak is. This is due to an efficient transfer of energy to surface plasmon. The decrease of the SPR peak width results in an increase of the reflectance for the propagating ray upon hydrogenation as shown in SPR curve. Since the rate $\Delta \epsilon_{2} / \Delta \epsilon_{1}$ is larger toward the long wavelengths, the change in reflectance is more significant near the infrared than the blue wavelength. For polarization $s$, the intensity decreases upon hydrogenation. It is explained by a more semiconductive behavior of the Pd film upon hydrogenation. The decrease is larger at shorter wavelengths since the amount of hydrogen seen by the probe light increases. With our sensor design, the sensor response is the sum of the $p$ and $s$ polarization light. Thus, the wavelength dependence of the sensor response come from the difference in reflectance with hydrogenation between polarization $s$ and $p$ depending on the wavelength. 


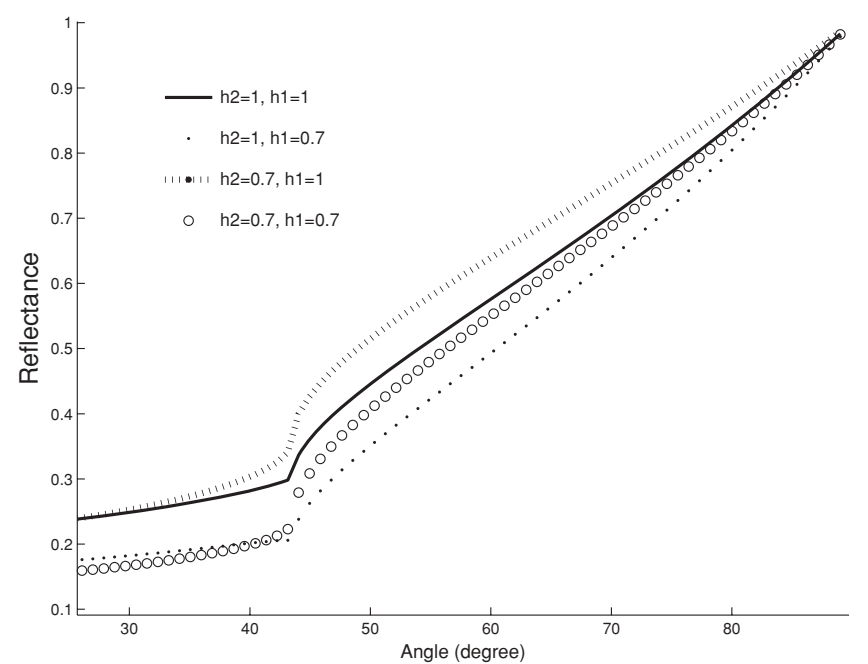

(b)

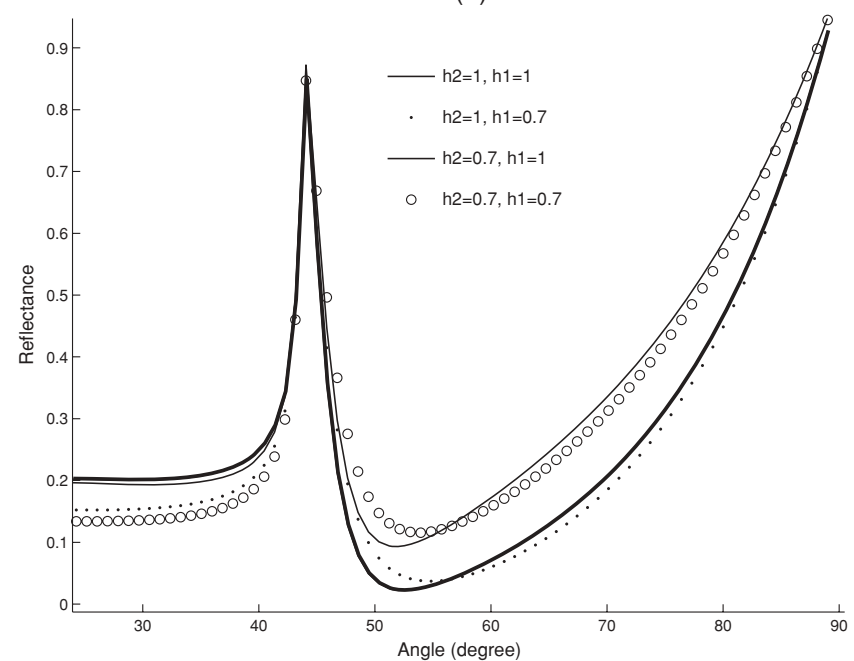

(a)

Fig. 9 Reflectance of a PdHx layer of $12 \mathrm{~nm}$ as a function of the angle $\theta$ (0 deg corresponds to the normal incidence) for a wavelength of $468 \mathrm{~nm}[(\mathrm{a}) p$ and (b) $s$ polarized].

It is worth noting that the sensitvity is higher when only one polarization is considered, as shown in Fig. 7. In a field test, it is difficult to maintain the polarization until the transducer region involves the use of polarization-controlling elements. The wavelength dependence is also related to the difference between the real and the imaginary part of the dielectric permittivity with hydrogenation. In order to reinforce this point, the dielectric permittivity of Pd hydride is described by the following relation:

$\epsilon_{\mathrm{Pd}, c(\%) \mathrm{H}_{2}}=h_{1}[c(\%)] \times \epsilon_{1 \mathrm{Pd}, 0 \% \mathrm{H}_{2}}+j h_{2}[c(\%)] \times \epsilon_{2 P d, 0 \% \mathrm{H}_{2}}$,

where $h_{1}$ and $h_{2}$ represent a nonlinear relation with the hydrogen concentration depending on the wavelength. Figure 9 shows the influence on each parameter on the reflectance. From the calculated reflectance, we infer that the reflectance for polarization $p$ strongly depends on $h_{2}$, whereas the reflectance for polarization $s$ depends more on the real part of the dielectric permittivity. In the case of Bevenot's sensor, ${ }^{8}$ relation (2) is satisfied because only polarization $p$ is considered. The imaginary part change is predominant on the

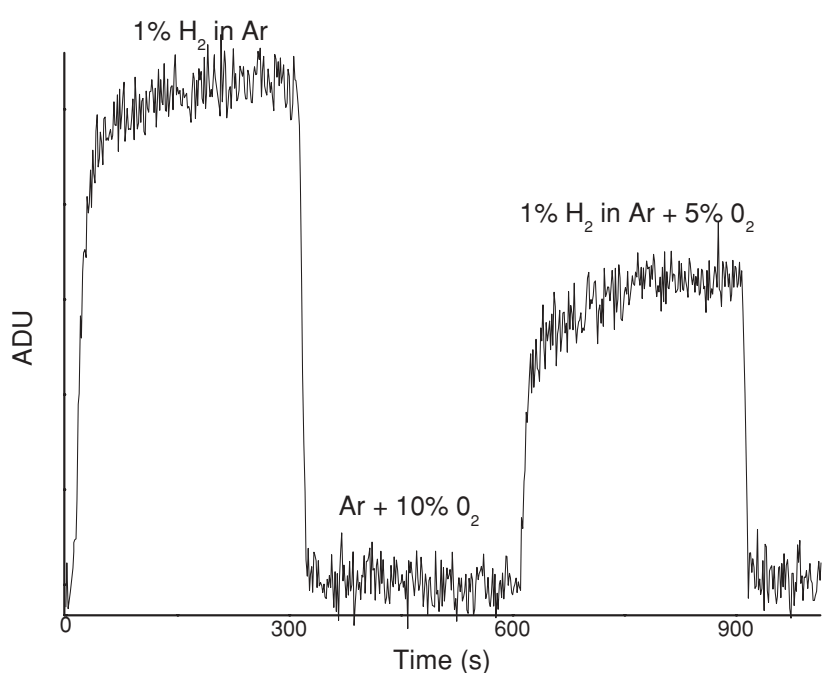

(a)

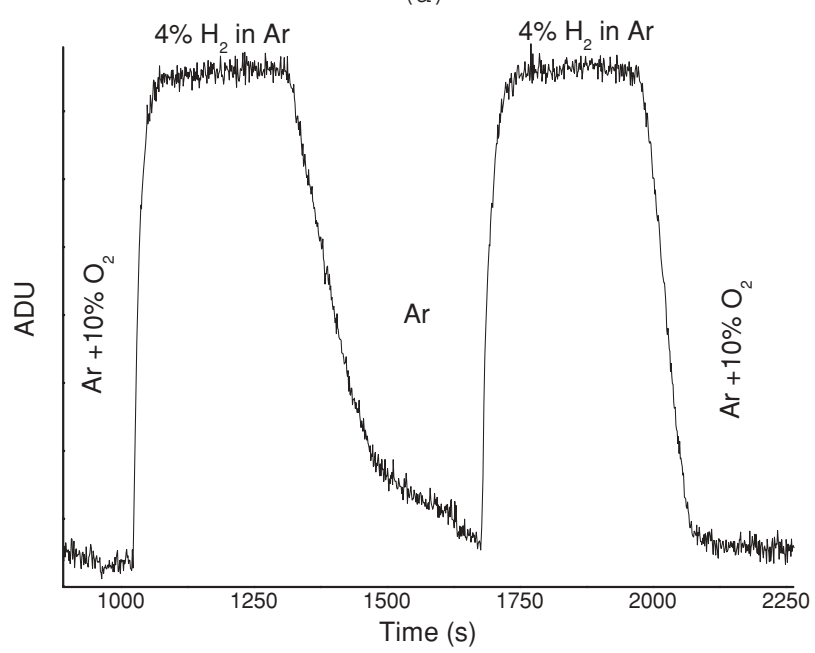

(b)

Fig. 10 The effect of $\mathrm{O}_{2}$ on the (a) loading and (b) unloading for a wavelength of $799 \mathrm{~nm}$.

response. So the constant $h$ experimentally determined in the previous work ${ }^{8}$ corresponds to the parameter $h_{2}$. For a spectral study, $h_{2}$ has to be determined for each wavelength. In our case, the relative transmission when only $p$ polarization is considered can be simulated by using relation (2) with $h=h_{2}=\epsilon_{2, \operatorname{Pd} H x} / \epsilon_{2, \mathrm{Pd}}$.

For safety application, the sensor is evaluated in an inert and in an oxygen environment. The loading time for both samples is $50 \mathrm{~s}$ for $4 \%$ of $\mathrm{H}_{2}$ in Ar during the first loading and decreases to $30 \mathrm{~s}$ after the first cycle when the recovery was done with a mix of $\mathrm{Ar}$ and $\mathrm{O}_{2}$. The presence of $\mathrm{O}_{2}$ during the recovery does not significantly influence the response signal level of the next loading time, but may increase the loading time. In fact, the loading time may decrease when the gas cell is flushed with $\mathrm{Ar}$ for a few minutes before cycling, in order to clean the Pd surface. This phenomenon is explained by the fact that the response time is mainly determined by the surface reaction and hydrogen diffusion. Since the $\mathrm{Pd}$ layer in our work is very thin, the catalytic reaction becomes the limiting step. We observe that the loading time can vary to 20 to $40 \mathrm{~s}$ during different cycling. We believe that the change of the response times are related to the 
surface reactions, which depend very strongly on the deposition technique used and the environmental conditions (temperature, pollutants, humidity, and avoidable sites on the surface). The loading time does not show a significant dependence on the $\mathrm{H}_{2}$ concentration compared to the previous ones. More oxygen during the loading can lead to a reduced sensitivity level. The response corresponding to a concentration of $1 \%$ of Hydrogen can decrease to one half of its value depending on the selected wavelength when a concentration of $5 \%$ of $\mathrm{O}_{2}$ was mixed with $\mathrm{Ar}$, as depicted in Fig. 10(a). The presence of $\mathrm{O}_{2}$ influences the absorption of $\mathrm{H}_{2}$ and is capable of limiting the amount of $\mathrm{H}_{2}$ absorped due to the formation of $\mathrm{OH}$ and $\mathrm{H}_{2} \mathrm{O}$ onto the $\mathrm{Pd}$ surface ${ }^{16}\left(\mathrm{H}_{2} \mathrm{O}\right.$ and $\mathrm{OH}$ will cost less energy than $\mathrm{H}_{2}$ absorption). The unloading time is longer than $150 \mathrm{~s}$, and can be decreased to $80 \mathrm{~s}$ by using $\mathrm{O}_{2}$ in the $\mathrm{Ar}$ flow as shown in Fig. 10(b). Oxygen on the surface of Pd will promote the formation of $\mathrm{OH}$ and $\mathrm{H}_{2} \mathrm{O}$ molecules, ${ }^{17}$ which significantly increases the desorption rate. The performance of such a sensor could be improved by adding a dopant in the Pd top-surface such as gold or copper to lower the sticking coefficient for $\mathrm{O}_{2} \cdot{ }^{18}$ A protective coating could be also used, but it should be choosen in order to avoid the extinction of the SPR, since it directly influences the confinement of the field into the metal. This protective layer could also enhance the SPR, for instance, by moving the SPR peak toward the angle within the NA fiber.

\section{Conclusion}

We report a wavelength dependence of the optical response on hydrogen loading of a Pd coated, fiber optic SPR based hydrogen sensor. The sensitivity of such a sensor can be enhanced by choosing a wavelength adapted to an optimal response. Furthermore, the wavelength dependent behavior of the SPR-hydrogen sensor allows one to monitor the response at several wavelengths which improves the hydrogen detection and avoids the generation of false hydrogen alarms by elimating temperature dependant shifts or mechanical vibration effects in the intensity of the measurement signal. The loading and unloading times are acceptable. An optimization of the Pd surface, matched with the SPR technique, would allow one to realize a low cost sensitive sensor with a fast response time.

\section{Acknowledgments}

The authors would like to thank the support given by Davide Ianuzzi's group at the VU University. We acknowledge financial support from the Nederlandse Organisatie voor Wetenschappelijk Onderzoek NWO through the Sustainable Hydrogen Programme of Advanced Chemical Technologies for Sustainability program. The funding from the Region Alsace in France is gratefully acknowledged.

\section{References}

1. Butler, "Optical fiber hydrogen sensor," Appl. Phys. Lett. 45, 10071009 (1984).

2. R. R. J. Maier, B. J. S. Jones, J. S. Barton, S. McCulloch, T. Allsop, J. D. C. Jones, and I. Bennion, "Fibre optics in palladium-based hydrogen sensing," J. Opt. A Pure Appl. Opt. 9, 45-59 (2007).

3. J. Villatoro and D. Monzón-Hernández, "Fast detection of hydrogen with nano fiber," J. Opt. Soc. Am. 13, 5087-5092 (2005).
4. B. Sutapun, M. Tabib-Azar, and A. Kazemi, "Pd-coated elastooptic fiber optic bragg grating sensors for multiplexed hydrogen sensing, "Sens. Actuators, B: Chemical 60, 27-34 (1999).

5. A. Trouillet, E. Marin, and C. Veillas, "Fiber grating for hydrogen sensing,"Meas. sci. technol. 17, 1124-1128 (2006).

6. C.-L. Tien, H.-W. Chen, W.-F. Liu, S.-S. Jyu, S.-W. Lin, and Y.-S. Lin "Hydrogen sensor based on side-polished fiber bragg gratings coated with thin palladium film,”. Thin Solid Films 516, 5360-5363 (2008), ISSN 0040-6090, thin Films 2006, Singapore.

7. J. Villatoro, D. Luna-Moreno, and D. Monzón-Hernández, "Optical fiber hydrogen sensor for concentrations below the lower explosive limit,"Sens. Actuators, B: Chemical 110, 23-27 (2005).

8. X. Bevenot, A. Trouillet, C. Veillas, H. Gagnaire, and M. Clement, "Surface plasmon resonance hydrogen sensor using an optical fibre," Meas. Sci. Technol. 13, 118-124 (2002).

9. B. Chadwick and M. Gal, "Enhanced Optical-Detection of Hydrogen Using the Excitation of Surface-Plasmons in Palladium", Applied Surface Science, 68 135-138 (1993).

10. E. Kretschmann, "The determination of the optical constants of metals by excitation of surface plasmons," Z. Phys. 241, 313-324 (1971)

11. F. Abeles, "La theorie generale des couches minces," J. Phys. Radium 11, 307-310 (1950)

12. B. Sullivan, "Optical properties of palladium in the visible and near uv spectral regions," Appl. Opt. 29, 1964-1968 (1990).

13. K. von Rottkay, M. Rubin, and P. A. Duine, "Refractive index changes of pd-coated magnesium lanthanide switchable mirrors upon hydrogen insertion," J. appl. phys. 85, 408-413 (1999).

14. W. Vargas, I. Rojas, D. Arzofeifa, and N. Clark, "Optical and electrical properties of hydrided palladium in thin films studied by an inversion approach from transmittance measurements," Thin Solid Films 496, 189-196 (2006)

15. Y. Yamada, K. Tajima, S. Bao, M. Okada, A. Roos, and K. Yoshimura, "Real time characterization of hydrogenation mechanism of palladium thin films by in situ spectroscopic ellipsometry," J. appl. phys. 106, 013523 (2009).

16. Z. Zhao, M. Knight, S. Kumar, E. T. Eisenbraun, and M. A. Carpenter, "Humidity effects on Pd/Au-based all-optical hydrogen sensors," Sens. Act. B: Chemical 129, 726-733 (2008).

17. L. G. Petersson, H. M. Dannetun, and I. Lundstrom, "Hydrogen detection during catalytic surface evidence for activated lateral hydrogen mobility in the water-forming reaction on pd," Phys. Rev. Lett. 52, 2 (1984).

18. M. Slaman, B. Dam, H. Schreuders, and R. Griessen, "Optimization of mg-based fiber optic hydrogen detectors by alloying the catalyst," Int J. Hydrogen Energy 33, 1084 - 1089 (2008).

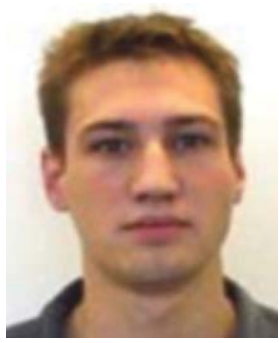

Cedric Perrotton is a PhD student in the school of Mathematics, Information and Engineering Sciences at the University of Strasbourg. He received his MS of Images, Sciences and Information Technologies, specialty photonics from the University of Strasbourg and his engineer master's degree from the Ecole Nationale Superieure de Physique de Strasbourg. His research interest is to develop hydrogen sensors based on optical fibers.

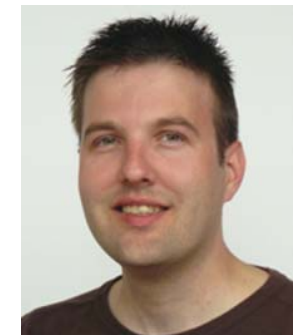

Martin Slaman graduated from the Hogeschool van Utrecht (Ing. degree) in The Netherlands, in 2001, in the field of electronics and computer science. The graduation project with the title characterization of III-V based near- and mid-infrared photovoltaic sensors was done in the institute of microelectronics IMEC in the Belgium city Leuven. As follow-up on this project the work on infrared detectors and camera systems continued until the end of 2001 within IMEC and then until May 2003 in the spin-off company XenICs. After this period he joined the Condensed Matter Group of VU University Amsterdam as a research engineer on the fiber optic hydrogen sensor project. The research in this project was focused on the use of metal hydridee switchable mirrors and optical fibers to detect hydrogen below the explosion limit. Currently he is working both for TU Delft and VU University Amsterdam in the fields of hydrogen detection, hydrogen purification membranes, fiber-optic micromachined devices, and solar-thermal collectors. 


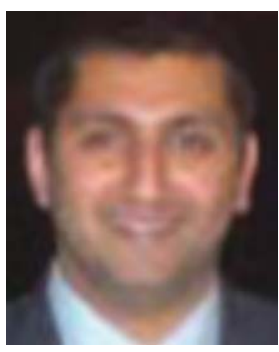

Nicolas Javahiraly received his $\mathrm{PhD}$ in photonics and laser physics from the University of Strasbourg (France) in 2002. After a postdoc at Harvard University where he worked on interaction laser matter (nano neuro laser surgery), he joined the Sagem Defense group in Paris. He worked there as a project manager doing research on night vision and new generation screens. He is an assistant professor at the University of Strasbourg. His current research interests include fiber optic sensors, fiber laser sources, and interaction laser matter.

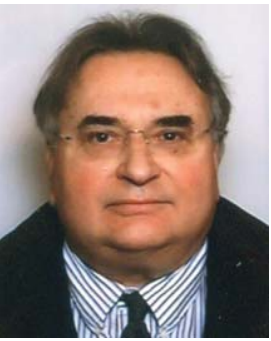

Patrick Meyrueis is a full professor at the University of Strasbourg. He is the founder and the head of the photonics system laboratory. He holds 200 publications and patents. $\mathrm{He}$ was the co-chairman of Photonics Europe SPIE at the beginning of this conference in Europe. His personal research concerns mainly photonics ecologic technology. $\mathrm{He}$ is also the founder of the Green Technology SPIE conference that will take place in Strasbourg in March 2011

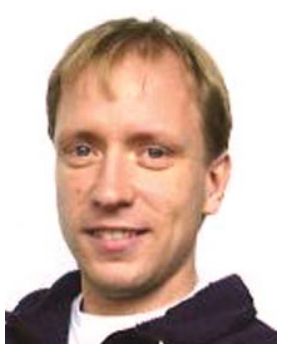

Herman Schreuders received his BSc in engineering physics from the Hogeschool Rijswijk. In 1995 he joined Philips Research in Eindhoven, The Netherlands. Within Philips he did research on cathode ray tubes, liquid crystal displays, and plasma displays. His main focus was on plasma discharges, electron transport via insulators, and high voltage insulation. In 2003, he started as an engineer in the Condensed Matter Physics Group of the Vrije Universiteit in Amsterdam. His main responsibility was sputter deposition of thin films for projects within the group. In 2009 the hydrogen research moved to the Materials for Energy Conversion and Storage (MECS) group in the Department of Chemical Engineering at Delft University of Technology where he continued work on hydrogen sensors, switchable mirrors, and lightweight hydrogen storage materials.

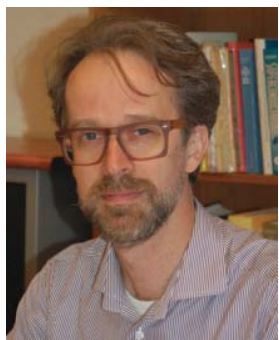

Bernard Dam received his PhD in 1986 for a thesis on the growth and morphology of incommensurate crystals. He spent six years at Philips Research Labs investigating the growth of thin film high-Tc superconductors and other functional complex oxides. From 1992 he worked at the VU Condensed Matter Department as an associate professor in the group of Prof. R. Griessen. After studying defect pinning in high-Tc superconductors, he turned to the field of metal hydrides. He developed a research line focused on the application of metal hydrides for sustainable energy applications, including hydrogen storage, sensors, membranes, etc., using a thin film combinatorial approach. Since 2009 he is the head of the MECS group at the Delft University of Technology, which focuses on Materials for Energy Conversion and Storage. In addition to the metal hydride research, this group specializes in photoelectrochemical water splitting process and the defect chemistry of oxide semiconductors. 